\section{Die Entziehung der ärztlichen Approbation}

Häufig sieht sich ein Arzt und gelegentlich ein Radiologe im Nachgang zu einem Strafverfahren einem Approbationsentziehungsverfahren vor der Approbationsbehörde ausgesetzt - jedenfalls dann, wenn das Strafverfahren mit einem Strafbefehl oder einem Strafe aussprechenden Urteil, also nicht durch einen Freispruch endet. Es muss gleichwohl nicht zwingend ein strafrechtliches Ermittlungsverfahren vorangehen, sofern die Approbationsbehörde Kenntnis von Umständen erlangt, die belegen, dass die Unwürdigkeit oder Unzuverlässigkeit zur Ausübung des ärztlichen Berufs eingetreten sein könnte. Besonders aktuell ist dabei die Frage, ob SARS-Cov-2leugnenden Ärzten die Approbation entzogen werden kann oder sogar muss. Die Approbationsbehörde ist weder die Ärztekammer noch ein ärztliches Berufsgericht, vielmehr handelt es sich dabei um auf Länderebene angesiedelte Verwaltungseinheiten. In einigen Ärztekammerbezirken können die ärztlichen Berufsgerichte jedoch die Feststellung eines zur Ausübung des Arztberufes unwürdigen Arztes treffen und damit mittelbar der Approbationsbehörde eine tatsächliche Grundlage für deren Einleitung eines Prüfverfahrens schaffen.

Nach $\S 5$ Abs. 2 S. 1 in Verbindung mit $\S 3$ Abs. 1 Nr. 2 Bundesärzteordnung (BÄO) ist die Approbation eines Arztes zu widerrufen, wenn der Arzt sich eines Verhaltens schuldig gemacht hat, aus dem sich seine Unwürdigkeit oder Unzuverlässigkeit zur Ausübung des ärztlichen Berufs ergibt. Darüber hinaus kann die Approbationsbehörde bei einer Erkrankung feststellen, dass der Arzt in gesundheitlicher Hinsicht nicht mehr zur Ausübung des Berufs geeignet ist. Die Ausübung der ärztlichen Tätigkeit ohne Approbation ist untersagt und wird sanktioniert.

\section{Feststellung der Unwürdigkeit nach der Bundesärzteordnung}

Der Widerruf der Approbation kann aufgrund der Unwürdigkeit oder Unzuverlässigkeit eines Arztes zur Ausübung der ärztlichen Tätigkeit erfolgen. Nach dem
Bundesverwaltungsgericht (BVerwG, Beschluss vom 09.01.1991, Az. 3 B 75/90) ist zwischen Unzuverlässigkeit und Unwürdigkeit wie folgt zu unterscheiden:

Der Begriff der „Unzuverlässigkeit” werde im Berufsrecht durch die Prognose gekennzeichnet, ob der Betroffene in Zukunft seine beruflichen Pflichten zuverlässig erfüllen werde (BVerwG, Buchholz $451.41 \S 15$ Nr. 3); „Unwürdigkeit“ liege nach Auffassung des Hessischen Verwaltungsgerichtshofs (Hess. VGH, Beschluss vom 04.03.1985, NJW 1986, 2390) bereits vor, wenn der Arzt durch sein Verhalten nicht mehr das zur Ausübung des ärztlichen Berufs erforderliche Ansehen und Vertrauen besitzt. Das BVerwG hält den Widerruf der Approbation wegen Unzuverlässigkeit nur dann für rechtens, wenn Tatsachen die Annahme rechtfertigen, der Arzt werde in Zukunft die berufsspezifischen Vorschriften und Pflichten nicht beachten; die Rechtfertigung des Widerrufs der Approbation wegen Unwürdigkeit sieht es in dem Ansehens- und Vertrauensverlust, hervorgerufen durch das zurückliegende Verhalten des Arztes. Bereits in seinem Beschluss vom 02.11.1992 (Az. 3 B 87/92) bestätigte das BVerwG diese Differenzierung und führte aus, dass, ob sich ein Arzt zur Ausübung des ärztlichen Berufs als „unwürdig“ erwiesen habe, nicht von der Prognose abhänge, ob der Arzt in Zukunft die beruflichen Pflichten zuverlässig erfüllen werde.

Über die Feststellung der Unwürdigkeit eines Arztes hatte zuletzt das Oberverwaltungsgericht (OVG) Lüneburg in seinem Beschluss vom 15.12.2020 (Az. 8 LA 80/20) zu entscheiden. Hintergrund des Verfahrens war der Widerruf der Approbation wegen des Verordnens von Benzodiazepinen ohne medizinische Indikation. Gegen den betreffenden Arzt war im Jahre 2016 wegen Untreue zulasten der Krankenkassen ein Strafbefehl über eine Geldstrafe in Höhe von 90 Tagessätzen ergangen. Die Tagessatzanzahl des Strafbefehls unterschritt damit die Grenze der Eintragung der Geldstrafe in das Führungszeugnis um exakt einen Tagessatz. Nach dem OVG Lüneburg bestehe Unwürdigkeit, wenn der Arzt ein Verhalten gezeigt habe, dass mit dem

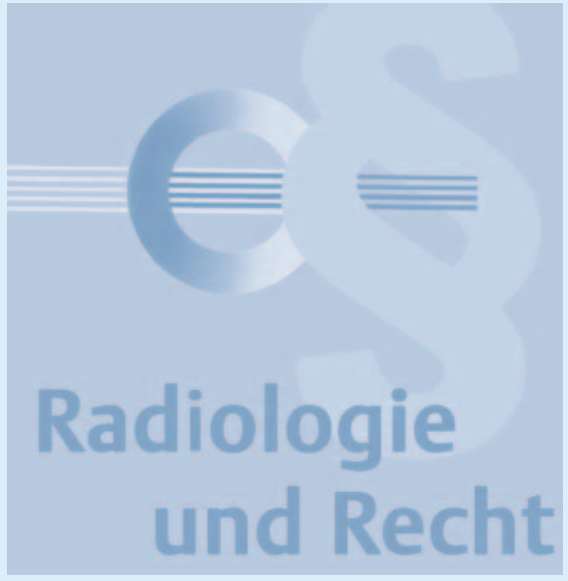

Berufsbild und den allgemeinen Vorstellungen von der Persönlichkeit eines Arztes schlechthin nicht zu vereinbaren sei, und er daher nicht mehr das Ansehen und Vertrauen genieße, das für die Ausübung des Berufes unabdingbar sei (BVerwG, Beschluss vom 31.07.2019, Az. 3 B 7/18). Anlass für den Approbationswiderruf könne mit Blick auf den grundgesetzlich gewährleisteten Schutz der Berufsfreiheit (Art. 12 Abs. 1 Grundgesetz) und das Verhältnismäßigkeitsgebot nur ein schwerwiegendes Fehlverhalten sein, das geeignet sei, das Vertrauen der Öffentlichkeit in den ärztlichen Berufsstand nachhaltig zu erschüttern. Dieses für das Arzt-PatientenVerhältnis konstitutive und für die Gesundheitsversorgung der Bevölkerung unerlässliche Vertrauen in die Integrität der Ärzteschaft würde durch eine fortdauernde Berufstätigkeit von Berufsträgern beeinträchtigt, die sich durch ihr Verhalten eines solchen Vertrauens unwürdig erwiesen haben. Nicht erforderlich sei, dass die gravierende Verfehlung auch strafbewehrt oder im konkreten Fall strafrechtlich geahndet worden sei.

In tatsächlicher Hinsicht können zur Feststellung der Unwürdigkeit die in vorangehenden Strafverfahren gewonnen Erkenntnisse und Beweismittel berücksichtigt werden. Es ist höchstrichterlich bestätigt, dass sowohl im Fall des Abschlusses eines Strafverfahrens durch Strafbefehl (BVerwG, Beschlüsse vom 18.08.2011, Az. 3 B 6.11 und 06.03.2003, Az. 3 B 10.03) wie auch bei der Verfahrenseinstellung nach $\S \S 153$, 153a StPO (BVerfG, Beschluss vom 16.01.1991, Az. 1 BvR 1326/90; Urteil vom 26.09.2002, Az. 3 C 37.01; Beschluss vom 
28.04.1998, Az. 3 B 174.97) eine eigenständige Überprüfung der in einem staatsanwaltschaftlichen Ermittlungsverfahren oder strafgerichtlichen Verfahren gewonnenen Erkenntnisse und Beweismittel im Hinblick auf das Vorliegen der Voraussetzungen des Approbationswiderrufs erfolgen kann. Zwar dürfe allein aus der Verfahrenseinstellung auf dieser Rechtsgrundlage, die nur mit Zustimmung des Angeschuldigten bzw. Angeklagten möglich sei, nicht auf die Verwirklichung des objektiven Tatbestandes der angeklagten Straftaten geschlossen werden. Die Ermittlungsergebnisse der Strafverfolgungsorgane dürfen jedoch von anderen Behörden und Gerichten in berufsrechtlichen Verfahren selbst ausgewertet, einer eigenständigen, nachvollziehbaren Bewertung unterzogen und auf dieser Grundlage eine berufsbezogene Zuverlässigkeitsprognose getroffen werden, so das OVG Lüneburg.

Im Rahmen der Prüfung, ob ein solcher Vertrauensverlust gegeben sei, dürfe, so das Verwaltungsgericht (VG) Hamburg in seinem Urteil vom 23.02.2019 (Az. $17 \mathrm{~K}$ 4618/18), kein idealisierendes Bild des Berufsstandes zugrunde gelegt werden. Der Approbationswiderruf diene nicht etwa dem Zweck, in der Öffentlichkeit den Eindruck einer „moralischen Makellosigkeit“ der Ärzteschaft aufrechtzuerhalten - auch ein approbierter Arzt unterliege nicht per se überhöhten moralischen Anforderungen. Er müsse keineswegs ein gleichsam „besserer Mensch“ als der empirische Durchschnittsbürger sein. Daher sei ein fehlsames Verhalten des Berufsangehörigen, welches von der Öffentlichkeit lediglich moralisch missbilligt werde, grundsätzlich kein hinreichender Grund für den massiven Grundrechtseingriff des Approbationswiderrufs (vgl. BVerfG, Beschluss vom 08.09.2017, Az. 1 BvR 1657/17). Anderes gelte nur und erst dann, wenn der Arzt sich ein Fehlverhalten habe zuschulden kommen lassen, das bei wertender Betrachtung das für die Behandlung von Patienten unerlässliche spezifische Vertrauensverhältnis nachhaltig zerstören müsse. Zu dessen Bewertung ist auf einen objektiven Maßstab abzustellen: Entscheidend sei das Werturteil, welches ein billig und gerecht Denkender bei Kenntnis der tatsächlichen Gegebenheiten des jeweiligen Einzelfalls also nicht etwa unter dem Einfluss einer möglicherweise tendenziösen Medienberichterstattung - über das zur Überprüfung stehende Verhalten des Arztes fällen würde. Sei danach dem betreffenden Arzt infolge seines Fehlverhaltens die für ein Vertrauensverhältnis zu seinen Patienten unerlässliche Integrität abzusprechen, sei das hohe Schutzgut der Gesundheit der Bevölkerung gefährdet, was allein den im Approbationswiderruf liegenden Grundrechtseingriff zu rechtfertigen vermöge. Die Wahrung dieses Schutzgutes erfordere es nämlich, dass sich Patienten jederzeit dem Arzt als ihrem allein fachlich-medizinischen Erwägungen verpflichtetem Helfer uneingeschränkt anvertrauen können. Hätten die Patienten jedoch Anlass, infolge eines schwerwiegenden Fehlverhaltens des Arztes an seiner grundlegenden ärztlichen Integrität zu zweifeln, liege es nahe, dass die Patienten davon abgehalten werden, im Bedarfsfall ärztliche Hilfe in Anspruch zu nehmen. Erst hieraus sei eine Gefährdung des hochstehenden Rechtsguts der Gesundheit der Bevölkerung herzuleiten. Bloßes Missfallen über bestimmte Verhaltensweisen im beruflichen oder gesellschaftlichen Umfeld des Arztes rechtfertigen den Grundrechtseingriff hingegen nicht.

Ähnlich hatte bereits der Verwaltungsgerichtshof (VGH) Baden-Württemberg in Mannheim (Beschluss vom 28.07.2003, Az. 9 S 1138/03) ausgeführt, dass den Vertretern der Heilberufe heute (Anm: im Jahr 2003) nicht mehr in jeder Beziehung eine integre Lebensführung als Berufspflicht auferlegt werde mit der Folge, dass ein Arzt, der sich eines Vermögensdelikts schuldig gemacht habe, das Ansehen seines Berufsstands grundsätzlich nicht so schwer schädige, dass er als unwürdig angesehen werden müsse. Unwürdigkeit sei vielmehr nur dann zu bejahen, wenn der Arzt vorsätzlich eine schwere, gemeingefährliche, gemeinschädliche oder gegen die Person gerichtete, von der Allgemeinheit besonders missbilligte, ehrenrührige Straftat begangen hat, die ein die Durchschnittsstraftat übersteigendes Unwerturteil enthalte und zu einer tief greifenden Abwertung seiner Persönlichkeit führe. Ergänzend wies aber der VGH Mannheim daraufhin, dass Straftaten nicht unmittelbar im Verhältnis Arzt-Patient angesiedelt sein müssten. Erfasst würden vielmehr auch alle berufsbezogenen, das heiße mit der eigentlichen ärztlichen Tätigkeit in nahem Zusammenhang stehende Handlungen, und ferner, abhängig von der Schwere des Delikts, auch Straftaten außerhalb des beruflichen Wirkungskreises.

Dem Fall des VGH Mannheim lag ein Sachverhalt zugrunde, in dem ein Arzt zum Nachteil seiner Kollegen missbräuchlich Zugangsdaten zu einem Bankkonto verwendet und Gelder einer Praxis auf ein eigenes Bankkonto transferiert hatte. Ein solcher schwerwiegender Vertrauensbruch gegenüber ärztlichen Kollegen verletze zum einen die berufsrechtlich geschuldete Kollegialität. Zum anderen führe dieser nicht allein zu einem Ansehens- und Vertrauensverlust innerhalb der Ärzteschaft, sondern entfalte zudem Außenwirkung gegenüber der weiteren Öffentlichkeit, die den Arzt für den ärztlichen Beruf als auf absehbare Zeit untragbar erscheinen lasse. Denn die Öffentlichkeit verlange von einem Arzt neben der fachlich beanstandungsfreien Behandlung seiner Patienten grundsätzlich auch die Einhaltung der sonstigen ärztlichen Berufspflichten (vgl. OVG Münster, NJW 2003, 1888). Diese Erwartung und das darin zum Ausdruck kommende Vertrauen in die Seriosität der Ärzteschaft wäre in hohem Maß beeinträchtigt, wenn ein Angehöriger dieser Berufsgruppe trotz der schwerwiegenden berufsbezogenen Verfehlungen und einer Verurteilung zu einer hohen Freiheitsstrafe weiter als Arzt tätig sein könne. Die Bevölkerung erwarte von einem Arzt, dass er einer anderen Person, insbesondere einem anderen Kollegen, nicht willentlich erheblichen Schaden zufügt (vgl. OVG Münster, NWVBI 2003, 233). Bereits die nur dessen individuellem Gewinnstreben dienende Berufsausübung eines Arztes betrachte die Öffentlichkeit für sich genommen kritisch. Sichere sich aber ein Arzt darüber hinaus durch die Begehung krimineller Taten im Rahmen seiner Berufsausübung eine Erwerbsquelle von gewisser Dauer und Umfang, sei das Ansehen der Ärzteschaft im Ganzen erheblich beschädigt. Hinzu komme, dass der Arzt auch Urkundenfälschungen begangen habe, unter fremden Namen aufgetreten sei und ärztliche Dokumente verändert habe. Der damit verbundene Ansehens- und Vertrauensverlust sei vor allem deshalb erheblich, weil dem Arzt auf Grund seines Berufs eine Vielzahl von Tätigkeiten überantwortet sei, bei 
denen er Urkunden, Gesundheitszeugnisse und sonstige Bescheinigungen ausstellen müsse und dürfe. Deshalb und gerade weil die ärztliche Tätigkeit weitgehend keiner Kontrolle unterliege, werde durch die Begehung von Urkundsdelikten das Ansehen des Arztes und seiner Kollegen selbst dann untergraben, wenn es sich nicht um Urkundsdelikte aus dem ärztlichen Bereich handele. Dies gelte erst recht, wenn ein Arzt ärztliche Zulassungsdokumente verfälscht.

Das OVG Lüneburg bewertete neben den dargestellten strafrechtlich relevanten Verhaltensweisen in der Vergangenheit auch bereits Mängel der Patientendokumentation als gravierendes Fehlverhalten. Ärzte seien durch §630f Bürgerliches Gesetzbuch (BGB) und §295 Fünftes Buch Sozialgesetzbuch (SGB V) sowie $\S 10$ der MusterBerufsordnung der Ärzte sowie ggf. als Vertragsärzte der gesetzlichen Krankenkassen nach §57 Bundesmantelvertrag Ärzte (BMV-Ä) verpflichtet, in der Patientenakte sämtliche aus fachlicher Sicht für die derzeitige und künftige Behandlung wesentlichen Maßnahmen und deren Ergebnisse aufzuzeichnen, insbesondere die Anamnese, Diagnosen, Untersuchungen, Untersuchungsergebnisse, Befunde, Therapien und ihre Wirkungen, Eingriffe und ihre Wirkungen, Einwilligungen und Aufklärungen sowie auch Arztbriefe von vor- und mitbehandelnden Ärzten. Die ärztliche Dokumentation diene dabei der Therapiesicherung und der Rechenschaftslegung. Sie hat die Aufgabe, das Behandlungsgeschehen aufzuzeichnen und dadurch eine sachgerechte therapeutische Behandlung zu gewährleisten sowie Ärzte, die einen Patienten weiterbehandeln, zu informieren. Die erforderlichen Aufzeichnungen dienen nicht allein der ärztlichen Gedächtnisstütze, sondern auch dem Interesse der Patienten, die grundsätzlich ein Recht auf Einsicht in die sie betreffende Patientenakte haben. Darüber hinaus ist die Dokumentation für die vertragsärztliche Tätigkeit von Bedeutung, um eine eventuelle Wirtschaftlichkeits- oder Plausibilitätsprüfung zu ermöglichen. Die Behandlungsdokumentation ist mithin für die Weiterbehandlung durch andere Ärzte, die Nachvollziehbarkeit der Behandlung und Aufklärung von Behandlungsfehlern sowie eine externe Kontrolle von erheblicher Bedeutung und stellt daher keine bedeutungslose Nebenpflicht der ärztlichen Tätigkeit dar, auch wenn die insoweit festgestellten Unregelmäßigkeiten nicht das gleiche Gewicht haben wie strafrechtliche Vorwürfe, insbesondere solche gegen die körperliche Integrität von Patienten.

Im Fall eines Abrechnungsbetruges zulasten der Patienten oder Krankenkassen stellen die Approbationsbehörden die Unwürdigkeit schnell fest, jedenfalls dann, wenn der Abrechnungsbetrug von einer gewissen Dauer und Umfang war. So führte das VG Hamburg (Urteil vom 09.10.2018, Az. 17 K $6716 / 17)$ aus, dass ein allgemeiner tiefgreifender Verlust des Vertrauens in die Integrität eines wegen Abrechnungsbetruges bestraften Arztes sich hingegen aus der von allen billig und gerecht Denkenden geteilten Erwägung ergeben könne, dass ein Arzt, der Betrugsstraftaten verübe, um sich wirtschaftliche Vorteile zu verschaffen, auf eine Weise von einem übersteigerten Erwerbsstreben beherrscht sei, die mit grundlegenden ethischen Vorstellungen schlechthin unvereinbar sei. Eine solche Wahrnehmung werde regelmäßig besonders nahe liegen, wenn das strafbare Verhalten in Zusammenhang mit der Berufsausübung stehe. Ein derart von der Erlangung unberechtigter wirtschaftlicher Vorteile geprägter Mensch werde nach dem Urteil jedes billig und gerecht Denkenden als jemand wahrgenommen werden, der sein Verhalten und damit auch seine ärztlichen Entscheidungen primär an wirtschaftlichen Motiven orientiere, statt vorrangig am Patientenwohl. In derartigen Fallkonstellationen sei daher typischerweise das für eine ordnungsgemäße Aufgabenerfüllung unabdingbare Vertrauen zwischen Arzt und Patient als nachhaltig zerstört anzusehen.

\section{Berücksichtigung eines nachträglichen Wohlverhaltens}

Nach der ständigen Rechtsprechung der Verwaltungsgerichtsbarkeit ist es möglich, dass ein Arzt die durch eine gravierende Verfehlung eingebüßte Berufsunwürdigkeit während des laufenden behördlichen Verfahrens über den Widerruf der Approbation wiedererlangt. Die Wiedererlangung der Würdigkeit setzt voraus, dass sich an der zum Widerruf führenden Sachlage nachweislich etwas zum Guten geändert hat, also der Arzt das für die Ausübung seines Berufes erforderliche Ansehen und Vertrauen zurückerlangt hat (BVerwG, Beschluss vom 15.11.2012, Az. 3 B 36.12; vom 23.07.1996, Az. 3 PKH 4.96). Durch den Approbationswiderruf wegen Unwürdigkeit soll nicht das bisherige Verhalten des Arztes durch eine zeitliche Verhinderung der Berufsausübung sanktioniert, sondern das Ansehen der Ärzteschaft in den Augen der Öffentlichkeit geschützt werden. Dies geschieht freilich nicht als Selbstzweck, sondern um das für jede Heilbehandlung unabdingbare Vertrauen der Patienten in die Integrität der Personen aufrecht zu erhalten, denen mit der Approbation die staatliche Erlaubnis zur selbständigen Ausübung der Heilkunde verliehen ist, und in deren Behandlung sich die Patienten vertrauensvoll begeben. Die Würdigkeit zur Ausübung des ärztlichen Berufs besteht daher erst dann wieder, wenn der Arzt das erforderliche Ansehen und Vertrauen zurückerlangt hat, mithin nach einer Gesamtwürdigung aller Umstände nicht mehr zu besorgen ist, dass dessen selbstständige Berufstätigkeit das Vertrauen der Öffentlichkeit in den Berufsstand nachhaltig erschüttern könnte (BVerwG, Beschluss vom 15.11.2012, Az. 3 B 36/12). Dies erfordert regelmäßig einen längeren Bewährungsprozess, der nach der Rechtsprechung des Oberverwaltungsgerichtes Lüneburg mit mindestens fünf Jahren bei gravierenden Verfehlungen außerhalb des beruflichen Wirkungskreises und mindestens acht Jahren bei gravierenden Verfehlungen im beruflichen Wirkungskreis anzusetzen ist (OVG Lüneburg, Beschlüsse vom 23.09.2015, Az. 8 LA 126/15 und 29.07.2015, Az. 8 ME 33/15). Maßgeblich für den Beginn der Bewährungsfrist ist der Zeitpunkt, in dem die zur Annahme der Berufsunwürdigkeit führenden gravierenden Verfehlungen durch den Betreffenden eingestellt worden sind, gleich ob dies auf einem freiwilligen Willensentschluss des Betreffenden oder auf einer Aufdeckung und Ahndung der Verfehlungen durch Dritte, insbesondere Strafverfolgungs- oder Approbationsbehörden beruht. 


\section{Fazit}

Bei einer ganzen Reihe von berufsrechtlichen und strafrechtlichen Verstößen droht eine Approbationsentziehung entweder aufgrund der festgestellten Unwürdigkeit oder Unzuverlässigkeit. In aller Regel bringt die Approbationsentziehung einen Radiologen in eine existenzielle wirtschaftliche Situation. Je näher ein Missverhalten im Zusammenhang mit der ärztlichen Berufsausübung steht, umso wahrscheinlicher wird die Einleitung eines Verfahrens zur Entziehung der Approbation. Die Wahrscheinlichkeit steigt sodann je mehr ein Patient vorsätzlich geschädigt oder gefährdet wird. Aber auch Verhalten am Rande oder außerhalb der ärztlichen Tätigkeit können als Grund für eine Entziehung genügen. Insbesondere Straftaten im Zusammenhang mit der Erstellung von falschen Urkunden und im weiteren Sinne dem Vortäuschen von Sachverhalten wirken sich dabei nachteilig aus, weil der Arzt zwar eine hochregulierte Tätigkeit ausübt, die tatsächlich aber wenig überwacht wird, so dass das Vertrauen in die Integrität des Arztes von essentieller Bedeutung ist. Das für die Behandlung von Patienten unerlässliche spezifische Vertrauensverhältnis darf nicht nachhaltig zerstört sein.

Abschließend soll der Fokus noch einmal auf die eingangs angesprochenen SARS-CoV-2leugnenden Ärzte fallen: In der Regel berufen sich diese auf das in Art. 5 Abs. 1 des Grundgesetzes garantierte Grundrecht auf Meinungsfreiheit. Zu unterscheiden ist dabei zwischen der Entäußerung von Meinungen und Tatsachen. Eine falsche Tatsachenbehauptung unterfällt nicht dem Schutz des Art. 5 Abs. 1 des Grundgesetzes. Dies zeigt sich besonders deutlich an dem Beispiel der Leugnung der Verbrechen des Nationalsozialismus der in §6 Abs. 1 des Völkerstrafgesetzbuches bezeichneten Art. Die Billigung, Verharmlosung oder Leugnung des Holocausts und die daraus folgende Störung des öffentlichen Friedens konnte, da es sich bei den Verbrechen des Nationalsozialismus um eine historische Tatsache handelt, nach $\S 130$ Abs. 4 des Strafgesetzbuches unter Strafe gestellt werden. Die Leugnung von SARS-CoV-2 ist nicht unter Strafe gestellt; nach den obigen Ausführungen kommt es bei der Feststellung der Unwürdigkeit oder Unzuverlässigkeit aber darauf nicht entscheidend an, ob das Verhalten eines Arztes strafbewehrt ist oder nicht, sondern z. B. ob sich Patienten jederzeit dem Arzt als ihrem allein fachlich-medizinischen Erwägungen verpflichtetem Helfer uneingeschränkt anvertrauen können. Haben sie jedoch Anlass, infolge eines schwerwiegenden Fehlverhaltens des Arztes an seiner grundlegenden ärztlichen Integrität zu zweifeln, liegt es nahe, dass die Patienten davon abgehalten werden, im Bedarfsfall ärztliche Hilfe in Anspruch zu nehmen. Ob oder wie ein SARSCoV-2-leugnender Arzt einen an SARS-CoV2 erkrankten Patienten behandelt oder behandeln kann, erscheint paradox, ist aber jedenfalls fraglich. Mit dieser und weiteren Fragestellung des konkreten Einzelfalles werden sich die Approbationsbehörden befassen müssen.

René T. Steinhäuser

Rechtsanwalt

Rechtsanwälte Wigge

Großer Burstah 42

20457 Hamburg

Telefon: (040) 3398705-90

Telefax: (040) 3398705-99

Internet: www.ra-wigge.de

E-Mail: kanzlei@ra-wigge.de 